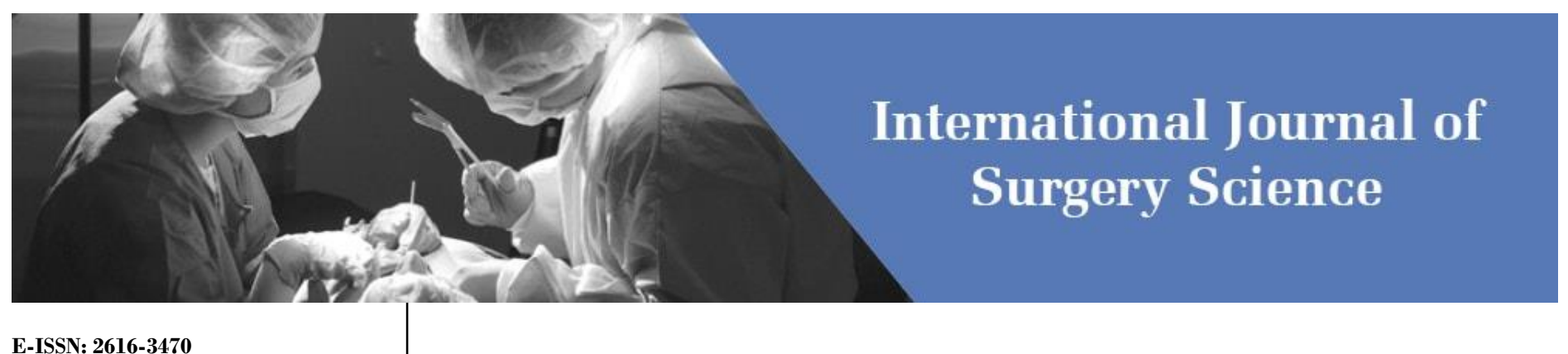

E-ISSN: 2616-3470

P-ISSN: 2616-3462

(C) Surgery Science www.surgeryscience.com

$2019 ; 3(3): 261-264$

Received: 04-05-2019

Accepted: 05-06-2019

Dr. Jamal Abed Hasan

Senior in General Surgery at AL-

Yarmouk Teaching Hospital,

Baghdad, Iraq

Dr. Qasim Hamza Eriby

Senior in General Surgery at AL

Yarmouk Teaching Hospital,

Baghdad, Iraq

Dr. Salih Abdul- Kafi Hammoodi Senior in General Surgery at ALYarmouk Teaching Hospital,

Baghdad, Iraq

\section{Causes of mortality in burns, prospective study}

\section{Dr. Jamal Abed Hasan, Dr. Qasim Hamza Eriby and Dr. Salih Abdul-Kafi Hammoodi}

DOI: https://doi.org/10.33545/surgery.2019.v3.i3e.178

\section{Abstract}

Background: Burn is a common emergency in clinical practice and remain a major medical and surgical problem.

Objectives: To diagnose the causes of death in patient who are burned with different types of burns.

Patients and Method: One hundred dead Patients were studied to diagnose the causes of death.

Results: Our study showed that 60 dead patients were females (60\%) and 40 dead patients were males (40\%) and female to male ratio were 1.5:1.The age ranged from 1 to 90 years, with mean age of the patients was 25 years \pm 5 years. our study showed that the most common cause of death were sepsis 50 (50\%) followed by multiorgan failure $25(25 \%)$, respiratory failure $15(15 \%)$, shock $8(8 \%)$, brain death 2 $(2 \%)$.

Conclusion: Our study showed that the most common cause of death were sepsis followed by multi organ failure, respiratory failure, shock and brain death.

Keywords: Study, death, shock, sepsis, brain, multiorgan

\section{Introduction}

Burn is a type of injury to flesh or skin caused by heat, electricity, chemicals, friction, or radiation ${ }^{[1]}$. Burn injury range from the trivial to sever burns that pose a threat to life, involve a long hospital stay, and carry the risk of permanent disfigurement or impaired function ${ }^{[2]}$.

The causes of burn may be due to

1. Thermal injury which are

a. Scald-spillage of hot liquids,

b. Flame burns,

c. Flash burns due to exposher of natural gas, alcohol, combustible liquids,

d. contact burns-contact with hot metals/objects/material,

2. Elictrical injury

3. Chemical burns (acid/alkali),

4. Cold injury-frost bite,

5. Ionising radiation,

6. Sun burns ${ }^{[3]}$.

\section{Burns may be classified into}

\section{Partial thickness burn which are:}

Erythema: A superficial burn with erythema due to capillary dilatation and with or without areas of blistering produce by exudation of plasma beneath coagulated epidermis, the underlying germinal layer is intact and complete healing takes place within a few days

Superficial partial thickness: Burn extends down through the epidermis to involve the germinal layer but the dermal appendages such as sweat gland and hair follicles remain largely preserved

Deep partial thickness: burn extends to the germinal layer and destroys a significant proportion of hair follicles, sebaceous glands and dermis.

2-Full thickness burns which there is completely destroy the skin ${ }^{[4]}$. 
Burns cause damage in a number of different ways, but by far the most common organ affected is the skin, however, burns can also damage the airway and lungs, with life-threatening consequences, airway injuries occur when the face and neck are burned, respiratory system injuries usually occur if a person is trapped in a burning vehicle, house, car or aero plane and is forced to inhale the hot and poisonous gases ${ }^{[5]}$. The characteristics of a burn depend upon its depth. Superficial burns cause pain lasting two or three days, followed by peeling of the skin over the next few days ${ }^{[6]}$. Individuals suffering from more severe burns may indicate discomfort or complain of feeling pressure rather than pain. Full-thickness burns may be entirely insensitive to light touch or puncture. While superficial burns are typically red in color, severe burns may be pink, white or black. Burns around the mouth or singed hair inside the nose may indicate that burns to the airways have occurred, but these findings are not definitive ${ }^{[7]}$. More worrisome signs include: shortness of breath, hoarseness, and stridor or wheezing. Itchiness is common during the healing process, occurring in up to $90 \%$ of adults and nearly all children ${ }^{[8]}$. Numbness or tingling may persist for a prolonged period of time after an electrical injury ${ }^{[9]}$. Burns may also produce emotional and psychological distress ${ }^{[10]}$.

\section{Patients \& Methods}

This is a prospective study of 100 death patients with Burn wounds were studied from January 2018to January 2019. Prior to death, all patients were admitted to the Hospitals and all were treated in an identical manner. Standard treatment included early excision of the burn wound, systemic and local antibiotic therapy, and continuous enteral and parenteral feeding.

\section{Results}

Our study showed that 60 dead patients were females $(60 \%)$ and 40 dead patients were males $(40 \%)$ and female to male ratio were 1.5:1. The age ranged from 1 to 90 years. with mean age of the patients was 25 years \pm 5 years. our study showed that the most common cause of death were sepsis $50(50 \%)$ followed by multiorgan failure $25(25 \%)$, respiratory failure 15 (15\%), shock $8(8 \%)$, brain death $2(2 \%)$ and, as shown in table 1 .

Table 1: Causes of death

\begin{tabular}{|c|c|c|}
\hline & The cause of death & Ratio \\
\hline 1 & sepsis & $50 \%$ \\
\hline 2 & Mutiorgan failure & $25 \%$ \\
\hline 3 & Respiratory failure & $15 \%$ \\
\hline 4 & shock & $8 \%$ \\
\hline 5 & Brain death & $2 \%$ \\
\hline & Total & $100 \%$ \\
\hline
\end{tabular}

\section{Discussion}

The mortality rate over this 20 -year review was $2.8 \%$. This is considerably lower than reported rates in the National Burn Repository $(5.6 \%)^{[11]}$. Low mortality rates, with high autopsy rates allowed us to investigate potential factors of clinical management that are correctable and could lead to improved survival. Acute lung injury or ARDS (Acute Respiratory Distress Syndrome) accounted for $40 \%$ to $50 \%$ of all deaths among the critically ill ${ }^{[12,13,14]}$. ARDS is a clinical diagnosis. Sixty-nine percent of patients that died from respiratory failure, died because of ARDS. Although the methods used for management of patients with ARDS has changed dramatically between 1989 and 1999 and 1999 and 2009, the mortality rate remained the same, whether or not there was clinical evidence of smoke inhalation injury. In addition, the breakdown of respiratory failure demonstrates the potential overlap of clinical diagnoses. Many patients that died of ARDS had evidence of pneumonia, and also demonstrated pathological evidence of DAD (Diffuse alveolar damage). Respiratory compromise can be a global problem in burn patients as mucus accumulates in distal parenchyma and bronchioles influenced by an increased secretory state of sub mucosal glands and decreased mucociliary function secondary to resuscitation efforts, and mechanical ventilation ${ }^{[15]}$. This physiologic change underscores the fact that overlapping diagnoses may contribute to death. Cases in which there were overlapping diagnoses, patients were placed in the category corresponding to the primary cause of death at autopsy. Thus, a patient may have pneumonia, but the primary cause of death was an anoxic brain injury. On the other hand, patients may have confirmation of anoxic brain injury at autopsy, but the primary cause of death was overwhelming sepsis. Patients diagnosed with ARDS were treated in concordance with the guidelines outlined in the ARDSNET trial in order to improve mortality ${ }^{[16]}$. Although this trial did not include pediatric burn patients, we had better outcomes with lower tidal volumes and lower plateau pressures ${ }^{[16]}$. The data suggest that the decrease in respiratory deaths from 1999 to 2009 may be associated with these gentler, supportive ventilator practices. Only $14 \%$ of deaths with burns were in patients with burns less than $40 \%$ of their TBSA (total body surface area) (minor burns). In addition, a quarter of patients that died from shock had burns encompassing less than $40 \%$ TBSA. These etiologies are attributed to delays in care or resuscitation, or deficient fluid resuscitation. Regardless of the extent of injury, airways for these particular patients were not obtained or maintained to ensure survival. A prior study looking at the determinants of mortality in severely burned patients underscored the relation between delays in resuscitation and increased mortality ${ }^{[17]}$. This study shows that this holds true despite the size of burn. Patients who died of sepsis had longer times until death compared with patients who died of other etiologies. This was significantly longer than in the anoxic brain injury, shock, and respiratory failure groups. Patients with respiratory failure had the second longest time until death, but this was not statistically significant. Life may have been prolonged in this patient population with the use of mechanical ventilation. Further investigation needs to be conducted to see if ARDS in this patient population was due, at least in part, to ventilator-associated pneumonia. Multi-organ failure was present after burn injury. It is caused here by sepsis, hypoxia, hypovolemia, and shock. Again, these etiologies can be attributed to delays and deficiencies in care and resuscitation. The most notable finding in this review was the effect of multidrug resistant organisms on long-term survival. From 1989 to 1999 , only $42 \%$ of patients died from sepsis from multi-drug resistant organisms and $25 \%$ of patients had Pseudomonas as the organism responsible. From 1999 to $2009,86 \%$ of patients that died from sepsis, died from multi-drug resistant organisms and 64\% of those patients had Pseudomonas as the organism responsible. Sepsis deaths from Acinetobacter did not arise in our institution until 1999 to 2009, and that organism was associated with the demise of $27 \%$ of patients with multi-drug resistant deaths. Although this was a substantial increase, it was not a statistically significant increase due to the sample size. Despite advances in anti-microbial therapies, the number of deaths associated with multiple antibiotic resistant organisms has increased. The incidence of invasive fungal infections decreased in the second decade. This finding is significant as invasive fungal infections lead to increased morbidity and 
mortality ${ }^{[18]}$. The decline in incidence of death due to fungal infection can be attributed to development of more effective antifungal therapies during the time period studied. In general, strategies to prevent infection, such as early excision and grafting, aggressive anti-microbial therapy, including the use of colistin, and early enteral feedings improve survival [19, 11, 15-20]. On the other hand, widespread use of aggressive anti-microbial therapies has led to increased colonization of pathogens that have resistance to current therapies ${ }^{[21,22]}$. In addition, faulty contact isolation practices propagate spreading the organism from one patient to the next ${ }^{[23,21]}$. With any signs of infection, patients were cultured, including blood, sputum, urine and tissue, and started on broad-spectrum antibiotics (covering for Gram-negative and Gram-positive organisms, fungi and parasites). Once cultures and sensitivities had been identified, therapy was tailored to these organisms. Despite these practices drug-resistant organisms remain a threat and challenge in the burn unit. The development and strengthening of pathogens to resist anti-microbial therapy are linked to the dramatic increase in the percentage of sepsis-related deaths in our institution. A recent study showed that female patients had a more attenuated hypermetabolic and inflammatory response compared with males ${ }^{[22]}$. Another issue raised by these findings relates to the aggressiveness with which we treat male and female patients. Female patients were more likely to die of respiratory failure, than any other cause, but had a lower incidence of inhalation injury. In addition, female patients had a lower incidence of sepsis. The question remains of whether female patients were more aggressively resuscitated, leading to fluid overload and need for mechanical ventilation, or if they received more aggressive anti-microbial therapy. In this study, all but one of all patients who died had an autopsy performed, thus, we suggest that these findings are representative of clinical care and management, despite the fact that autopsies are known to disagree with clinical diagnoses in up to $40 \%$ of cases [24, 25]. Patients, regardless of burn size, age, or point of origin have become more likely to survive a burn injury during the past 20 years. Those that did not survive had some evidence of delays or deficits in resuscitation with either airway management or volume leading to burn shock. The progression to multi-organ failure from shock was prolonged due to the extensive physiologic reserve and cardiac resilience that are characteristic of children. The development of sepsis significantly contributed to the demise of patients with and without the emergence of multi-drug resistant organisms. The main focus of this study was on the single primary immediate cause of death. Burn trauma is a complicated injury that causes profound physical and physiologic derangements. The clinical course for these patients is also complicated. For example, many patients died with anoxic brain injury but that injury was not the primary cause of death. Furthermore, many patients died with burn wound infections due to multi-resistant organisms, but these infections were not the primary cause of death. Some patients died with derangements in multiple organ systems, which led to their demise.

\section{Conclusion}

Our study showed that the most common cause of death were sepsis followed by multi organ failure, respiratory failure, shock and brain death.

1. Recommendation: A significant proportion of burns can be prevented by: 1-Implementing good health and safety regulations, 2-Educating the public,

\section{References}

1. Herndon D. Total burns care (4th ed.), Prevention of Burn Injuries, Edinburgh; Saunders, 2012, 46.

2. James D Watson. Principles and practice of surgery, $6^{\text {th }}$ edition, chrchill Livingstone Elsevier, 2012, 303.

3. Sriram Bhat M. SRBS Manual of surgery, $4^{\text {th }}$ edition, Jaypee brother medical publishers (p) LTD, 2013, 130.

4. Harold Ellis, Sir Roy Calne, Christover Watson. General surgery, lecture notes, $12^{\text {th }}$ edition, Wiley-Blackwell, 2010, 41.

5. Michael Tyler and Sudip Ghosh, Bailey \& Loves, short practice of surgery, $26^{\text {th }}$ edition, CRC Press, Taylor \& Francis Group, 2013, 385.

6. Granger Joyce. Pediatric Emergency Medicine Practice. An Evidence-Based Approach to Pediatric Burns, 2009.

7. Brunicardi Charles. Schwartz's principles of surgery, $9^{\text {th }}$ edition. New York, McGraw-Hill, Medical Pub Division, Chapter, 2010, 8.

8. Goutos I, Dziewulski P, Richardson PM. Pruritus in burns, review article, Journal of burn care \& research, official publication of the American Burn Association. 2009-2010; 30(2):221-8.

9. Marx John. Rosen's emergency medicin, concepts and clinical practice, $7^{\text {th }}$ edition, Philadelphia, Mosby/Elsevier, Chapter 140, Electrical and Lightning Injuries, 2010.

10. Peck MD. Journal of the International Society for Burn Injuries. Epidemiology of burns throughout the world, Part I: Distribution and risk factors, 2010-2011; 37(7):1087-100.

11. Miller SF, Bessey PQ, Schurr MJ, Browning SM, Jeng JC, Caruso DM, et al. National Burn Repository 2005: A tenyear review. J Burn Care Res. 2006; 27:411-436. Doi: 10.1097/01.BCR.0000226260.17523.22.

12. Greenhalgh DG, Saffle JR, Holmes JHt, Gamelli RL, Palmieri TL, Horton JW, et al. American Burn Association consensus conference to define sepsis and infection in burns. J Burn Care Res. 2007; 28:776-790. Doi: 10.1097/BCR.0b013e3181599bc9.

13. Jeschke MG, Chinkes DL, Finnerty CC, Przkora R, Pereira $\mathrm{CT}$, Herndon DN. Blood transfusions are associated with increased risk for development of sepsis in severely burned pediatric patients. Crit Care Med. 2007; 35:579-583. Doi: 10.1097/01.CCM.0000253812.09236.98.

14. Suman OE, Spies RJ, Celis MM, Mlcak RP, Herndon DN. Effects of a 12-wk resistance exercise program on skeletal muscle strength in children with burn injuries. J Appl Physiol. 2001; 91:1168-1175.

15. Cox RA, Mlcak RP, Chinkes DL, Jacob S, Enkhbaatar P, Jaso J, et al. Upper airway mucus deposition in lung tissue of burn trauma victims. Shock. 2008; 29:356-361. Doi: 10.1097/SHK.0b013e31814541dd.

16. The Acute Respiratory Distress Syndrome Network. Ventilation with lower tidal volumes as compared with traditional tidal volumes for acute lung injury and the acute respiratory distress syndrome. N Engl J Med. 2000; 342:1301-1308. Doi: 10.1056/NEJM200005043421801.

17. Wolf SE, Rose JK, Desai MH, Mileski JP, Barrow RE, Herndon DN. Mortality determinants in massive pediatric burns. An analysis of 103 children with $>$ or $=80 \%$ TBSA burns $(>$ or $=70 \%$ full-thickness) Ann Surg. 1997; 225:554-569. Doi: 10.1097/00000658-199705000-00012.

18. Murray CK, Loo FL, Hospenthal DR, Cancio LC, Jones JA, $\mathrm{Kim} \mathrm{SH}$, et al. Incidence of systemic fungal infection and related mortality following severe burns. Burns. 2008; 34:1108-1112. Doi:10.1016/j.burns.2008.04.007. 
19. Mandal RV, Mark EJ, Kradin RL. Organizing pneumonia and pulmonary lymphatic architecture in diffuse alveolar damage. Hum Pathol. 2008; 39:1234-1238. Doi: 10.1016/j.humpath.2008.01.002.

20. Rosanova M, Epelbaum C, Noman A, Villasboas M, Alvarez V, Berberian G, et al. Use of colistin in a pediatric burn unit in Argentina. J Burn Care Res. 2009; 30:612-615. Doi: 10.1097/BCR.0b013e3181abffb6.

21. Roberts MC. Antibiotic toxicity, interactions and resistance development. Periodontol. 2000-2002; 28:280-297. Doi: 10.1034/j.1600-0757.2002.280112.x.

22. Jeschke MG, Mlcak RP, Finnerty CC, Norbury WB, Przkora R, Kulp GA, et al. Gender differences in pediatric burn patients: does it make a difference? Ann Surg. 2008; 248:126-136. Doi: 10.1097/SLA.0b013e318176c4b3.

23. Roberts JA, Kruger P, Paterson DL, Lipman J. Antibiotic resistance-what's dosing got to do with it? Crit Care Med. 2008; 36:2433-2440. Doi: 10.1097/CCM.0b013e318180fe62.

24. Goldman L, Sayson R, Robbins S, Cohn LH, Bettmann M, Weisberg $\mathrm{M}$. The value of the autopsy in three medical eras. N Engl J Med. 1983; 308:1000-1005.

25. Mort TC, Yeston NS. The relationship of pre mortem diagnoses and post mortem findings in a surgical intensive care unit. Crit Care Med. 1999; 27:299-303. Doi: 10.1097/00003246-199902000-00035. 